\title{
ON THE INTEGRAL REPRESENTATION OF THE ENDOMORPHISMS OF MIKUSINŚKI'S OPERATOR FIELD
}

\author{
by ANDRÁS BLEYER $\dagger$ \\ (Received 19th March 1971)
}

\section{Introduction}

We proved in (1) that every continuous endomorphism can be generated on a subring of the field $M$. More precisely, the ring $H$ of piecewise polynomial functions has the property that every isomorphism from $H$ into $M$, continuous in the sequential topology of $H$, can be extended to a continuous endomorphism of $M$ where the notion of continuity in $M$ is the usual sequential one.

Gesztelyi (7) studied integral representations of linear transformations of the operator field and he proved that every continuous operator transformation which is continuous in his sense can be realized on the set of continuous functions as an integral transformation. But to decide whether an operator transformation is continuous in Gesztelyi's sense is either very difficult or cannot be carried out at all. The case when the operator transformation is multiplicative, i.e. is an endomorphism, is not any simpler.

In the applications there are several integral transformations whose Laplace transforms are more easily handled but it is not yet known whether these transformations are continuous in the sense of Gesztelyi. Since the unique extension is more important than continuity we do not lose too much if we weaken the above notion of continuity. This is the purpose of the present paper.

Throughout the first part we shall deal with a convergence that is weaker than that used in papers of Gesztelyi (7) and the author (1) but is strong enough for the extension theorem proved in (1). In the second part we give a new proof of Gesztelyi's representation theorem, and our theorem is a generalization of that in a certain sense; we prove the same statement under the condition of pointwise continuity assuming that $\mathscr{F}(s)$, the $\mathscr{F}$-map of the operator of differentiation, is a logarithm in the sense of Mikusinśki.

Finally we remark that in the construction of the operator field we start with the set of continuous functions with left bounded support. The operator field generated by the above set is isomorphic to the original operator field defined by Mikusinśki.

For this it is enough to show that every operator generated by functions with

$\dagger$ This paper was written while I was a Postdoctoral Fellow at the University of Edinburgh on a grant awarded by the Hungarian Academy of Sciences. 
left bounded supports can be generated by functions of $\mathscr{C} \dagger$. Indeed, let $x$ be an operator generated by functions $f$ and $g$ with left bounded supports. Then for some real numbers $\alpha$ and $\beta, f e^{-\alpha s}, g e^{-\beta s} \in \mathscr{C}$. But

$$
x=\frac{f}{g}=\frac{f e^{-(\alpha+\beta) s}}{g e^{-(\alpha+\beta) s}}=\frac{l f e^{-(\alpha+\beta) s}}{l g e^{-(\alpha+\beta) s}}
$$

and $l f e^{-(\alpha+\beta) s}, l g e^{-(\alpha+\beta) s}$ are functions from $\mathscr{C}$.

I. $\tau$-convergence and $\tau$-continuity

Definition 1.1. The sequence of continuous functions $p_{n}$ is $\tau$-convergent to

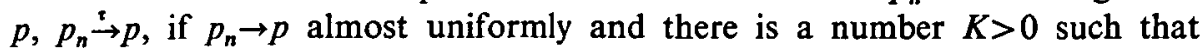
$\left|p_{n}(t)\right|<K \max \{|p(t)|, 1\}$ for each $n$ and for all $0 \leqq t<\infty$.

Definition 1.2. The sequence of operators $p_{n}\left(p_{n} \in M\right)$ is $\tau$-convergent to $p(p \in M), p_{n} \stackrel{\mathscr{b}}{\rightarrow} p$, if there is a function $q$ of $\mathscr{C}$ such that $q p_{n} \stackrel{\sim}{\rightarrow} q p$ in $\mathscr{C}$.

Definition 1.3. A linear operator transformation $\mathscr{F}: M_{1} \rightarrow M\left(M_{1}\right.$ is a subspace of $M$ ) is $\tau$-continuous if $p_{n} \stackrel{\mathscr{x}}{\rightarrow} p$ always implies $\mathscr{F}\left(p_{n}\right) \stackrel{M}{\rightarrow} \mathscr{F}(p)$ where $\stackrel{M}{\rightarrow}$ is the usual convergence (the Mikusinski convergence) in $M$.

Definition 1.4. A linear operator transformation $\mathscr{F}: M_{1} \rightarrow M$ is said to be $M_{1}$-strongly continuous if the operator function $\mathscr{F}(f(\lambda))$ is continuous whenever the operator function $f(\lambda)$ is continuous on $a \leqq \lambda \leqq b$ and has values in $M_{1}$.

This definition coincides with Gesztelyi's definition (7). The proofs of the following Theorems, 1.1 to 1.5, are similar to proofs given by Gesztelyi and will not be repeated here.

Theorem 1.1. Every $M_{1}$-strongly continuous linear operator transformation is continuous (in the sense of Bleyer (1) i.e. pointwise continuous) and hence is $\tau$-continuous.

Theorem 1.2. If $u \in \mathscr{C} \mathscr{U}$ then

$$
u=\int_{-\infty}^{\infty} u(\lambda) \exp (-\lambda s) d \lambda
$$

It is a well-known fact that the Mikusinśki integral holds for every locally integrable function with left bounded support. Hence we obtain a stronger form of Theorem 1.2 for $H$ :

for all $u \in H$.

$$
u=\int_{-\infty}^{\infty} u(\lambda) \exp (-\lambda s) d \lambda
$$

We shall use Stieltjes type integrals of operator functions defined by Gesztelyi $(6,7)$ for the integral representation of an operator transformation.

$\dagger f \in \mathscr{C}$ if and only if $f$ is continuous on $[0, \infty)$ and $f \equiv 0$ on $(-\infty, 0)$.

$\ddagger \mathscr{C} \mathscr{Q}=\left\{f: f\right.$ is continuous on $\left[\alpha_{f}, \infty\right)$ and $f=0$ on $\left(-\infty, \alpha_{f}\right)$ for some $\left.\alpha_{f}>-\infty\right\}$. 
Theorem 1.3. If $f(\lambda)$ is an operator function which is integrable with respect to $\phi(\lambda)$ in the interval $[\alpha, \beta], f(\lambda) \in[H]$ and $\mathscr{F}$ is an $[H]$-strongly continuous linear operator transformation then $\dagger \mathscr{F}(f(\lambda))=g(\lambda)$ is an integrable operator function and

$$
\int_{\alpha}^{\beta} \mathscr{F}(f(\lambda)) d \phi(\lambda)=\mathscr{F}\left(\int_{\alpha}^{\beta} f(\lambda) d \phi(\lambda)\right) .
$$

The theorem is valid for infinite intervals, but in that case we have to assume the convergence of the improper integral $\int_{\alpha}^{\beta} f(\lambda) d \phi(\lambda)$. If $f(\lambda)$ is a differentiable operator function in $(\alpha, \beta)$ then $\mathscr{F}(f(\lambda))$ is also differentiable and

$$
\frac{d}{d \lambda} \mathscr{F}(f(\lambda))=\mathscr{F}\left(\frac{d f(\lambda)}{d \lambda}\right) .
$$

Theorem 1.4. Every $[H]$-strongly continuous linear operator transformation of $[H]$ into $M$ can be written in the form

for all $u \in H . \ddagger$

$$
\mathscr{F}(u)=\int_{-\infty}^{\infty} u(\lambda) \mathscr{F}(\exp (-\lambda s)) d \lambda
$$

Theorem 1.5. If $\mathscr{F}$ is an [H]-strongly continuous isomorphism of $[H]$ into $M$, then $\mathscr{F}(s)=(\mathscr{F}(l))^{-1}$ is a logarithm and

for every $u \in H$.

$$
\mathscr{F}(u)=\int_{-\infty}^{\infty} u(\lambda) \exp (-\lambda \mathscr{F}(s)) d \lambda
$$

We proved the following theorem in (1).

Theorem 1.6. Let $\mathscr{C}_{1}$ be an arbitrary subalgebra of $M$ containing $H$ and let $\mathscr{F}$ be a continuous isomorphism of $\mathscr{C}_{1}$ into $M$. Then $\mathscr{F}$ can be uniquely extended to a continuous endomorphism of $M$.

The theorem is also true when we consider $\mathscr{F}$ as a $\tau$-continuous mapping. The proof is the same as before. (The results used for the proof of Theorem 1.6 can be found in papers of Boehme (2) and Mikusinśki (10).) Theorem 1.6 gives an unambiguous description of the continuous endomorphisms. In fact, $\mathscr{F}(s)$ is a logarithm by virtue of Theorem 1.5 for every [H]-strongly continuous endomorphism $\mathscr{F}$ and it follows from Theorem 1.1 that every $[H]$-strongly continuous endomorphism is also continuous, whence it is $\tau$-continuous too.

The truth of the converse statement is unknown. However, it seems unlikely that the converse statement is true unless we make further restrictions.

The following theorem will show how the integral representation of Theorem 1.5 is useful to describe $\tau$-continuous endomorphisms of $M$. But the complete

$\dagger[H]$ is the field of operators generated by $H$. Evidently $[H] \subset M$.

$\ddagger$ It is easy to see that $e^{-\lambda s} \in[H]$.

$$
\text { E.M.S. }-\mathrm{Z}
$$


answer to the question when there exists the integral representation of Theorem 1.5 is not yet known.

Theorem 1.7. (a) If $\mathscr{F}$ is an $[H]$-strongly continuous isomorphism of $H$ into $M$ then

exists for all $\alpha>-\infty$.

$$
\int_{a}^{\infty} \exp (-\lambda \mathscr{F}(s)) d \lambda
$$

(b) If $\int_{\alpha}^{\infty} \exp (-\lambda w) d \lambda$ is absolutely convergent for all $\alpha>-\infty$, i.e., if there is a continuous function $g$ of $\mathscr{C}$ such that $g \exp (-\lambda w) \in \mathscr{C}$ for all $\lambda>-\infty$ and the integral

$$
\int_{a}^{\infty}|g \exp (-\lambda w)| d \lambda
$$

is convergent for all $\alpha>-\infty$ then there exists only one $\tau$-continuous endomorphism $\widetilde{\mathscr{F}}$ of $M$ for which $w=\tilde{\mathscr{F}}(s)$ and

for all $u \in H$.

$$
\tilde{\mathscr{F}}(u)=\int_{-\infty}^{\infty} u(\lambda) \exp (-\lambda w) d \lambda
$$

Proof. Part (a) is an obvious consequence of Theorem 1.5. For part (b), if the integrals (1.7.1) exist then they exist for all $u \in H$ since every $u \in H$ can be written either in the form $u=\sum_{i=1}^{n} c_{i} \mathscr{H}_{\alpha_{i}}(t)$ or as a convolution product of a finite number of functions of this type. However the integral commutes with convolution and if $u=u_{n} * u_{m}$ then $\int_{-\infty}^{\infty} u(\lambda) \exp (-\lambda w) d \lambda$

$$
=\left[\int_{-\infty}^{\infty} u_{n}(\lambda) \exp (-\lambda w) d \lambda\right] *\left[\int_{-\infty}^{\infty} u_{m}(\lambda) \exp (-\lambda w) d \lambda\right] .
$$

Consequently the operator transformation $\mathscr{F}(u)=\int_{-\infty}^{\infty} u(\lambda) \exp (-\lambda w) d \lambda$ is a homeomorphism of $H$ into $M$. Now we show that $\mathscr{F}(u)=0$ implies $u=0$, i.e. $\mathscr{F}$ is, in fact, an isomorphism. To prove this, let us suppose that

$$
\int_{-\infty}^{\infty} \mathscr{H}_{\alpha_{0}}(\lambda) \exp (-\lambda w) d \lambda=0
$$

for some $\alpha_{0}>-\infty$. Then for all $\alpha_{1}>\alpha_{0}$

$$
-\int_{\alpha_{0}}^{\alpha_{1}} \mathscr{H}_{\alpha_{0}}(\lambda) \exp (-\lambda w) d \lambda=\int_{\alpha_{1}}^{\infty} \mathscr{H}_{\alpha_{0}}(\lambda) \exp (-\lambda w) d \lambda
$$


i.e.

$$
\frac{1}{w}\left[\exp \left(-\alpha_{1} w\right)-\exp \left(-\alpha_{0} w\right)\right]=\int_{\alpha_{1}}^{\infty} \exp (-\lambda w) d \lambda
$$

The right-hand side of this equation is uniformly convergent to zero in every interval $[0, T]$ as $\alpha_{1} \rightarrow \infty$. The left-hand side is convergent to

$$
-\frac{1}{w} \exp \left(-\alpha_{0} w\right) \neq 0
$$

since $\exp \left(-\alpha_{1} w\right) \rightarrow 0$ as $\alpha_{1} \rightarrow \infty$ follows from the absolute convergence of the integral (1.7.1). So for any $\alpha_{0}>-\infty$

$$
\int_{-\infty}^{\infty} \mathscr{H}_{\alpha_{0}}(\lambda) \exp (-\lambda w) d \lambda \neq 0
$$

Let us note that the integral (1.7.4) is equal to the integral (1.7.1) with $\alpha=\alpha_{0}$. Moreover, it is easy to verify that for every finite number $n$ the function system $\left\{\int_{-\infty}^{\infty} \mathscr{H}_{\alpha_{i}}(\lambda) \exp (-\lambda w) d \lambda\right\}_{i=1}^{n}$, for any pairwise different $\alpha_{i}$, is a linearly independent function system. Hence we have that if $u=\sum_{i=1}^{n} y_{i} \mathscr{H}_{\alpha_{i}}(\lambda) \neq 0$ then $\mathscr{F}(u) \neq 0$. Thus by virtue of the multiplicative property of $\mathscr{F}, \mathscr{F}(u)=0$ if and only if $u=0$.

Now we show that $\mathscr{F}$ is a $\tau$-continuous isomorphism of $H$ into $M$, so that by Theorem 1.6 it can be extended to the whole of $M$ uniquely.

Let $u_{n} \stackrel{\mathfrak{r}}{\rightarrow} u$ in $H$. Let us choose the numbers $r_{k}$ so that

$$
\int_{r_{k}}^{\infty}\left|u^{*}(\lambda) \| g \exp (-\lambda w)\right| d \lambda<\varepsilon_{k}(t)
$$

where $\left|u^{*}(\lambda)\right|=\max \{\mid u(\lambda) ; 1\}, \varepsilon_{k}(t)$ is an arbitrary sequence almost uniformly convergent to zero, and $g$ is a continuous function for which

$$
g \exp (-\lambda w)=\left\{g_{1}(\lambda, t)\right\}
$$

is a continuous function of both variables and $g$ is as in Theorem 1.7(b). From the fact that $u_{n} \dot{b} u$ it follows that the supports of these functions are bounded below, i.e. there is a common lower bound $\alpha$ say. Let the maximum of $\left\{\left|g_{1}(\lambda, t)\right|\right\}$ on the set $\left[\alpha, r_{k}\right] *[0, T]$ be denoted by $K\left(r_{k}, T\right)$. Choose $n_{0}$ so that for all $n \geqq n_{0}$

$$
\left|u_{n}(\lambda)-u(\lambda)\right|<\frac{\varepsilon_{k}}{\left(r_{k}-\alpha\right) K\left(r_{k}, T\right)}
$$

for all $\lambda \in\left[\alpha, r_{k}\right]$ where $\varepsilon_{k}=\max _{t \in[0, r]}\left|\varepsilon_{k}(t)\right|$. Then $\left|g \int_{\alpha}^{\infty}\left(u_{n}(\lambda)-u(\lambda)\right) \exp (-\lambda w) d \lambda\right| \leqq\left|\int_{a}^{r_{k}}\left(u_{n}(\lambda)-u(\lambda)\right) g \exp (-\lambda w) d \lambda\right|$ 


$$
\begin{aligned}
& +\left|\int_{r_{k}}^{\infty}\left(u_{n}(\lambda)-u(\lambda)\right) g \exp (-\lambda w) d \lambda\right| \leqq \int_{\alpha}^{r_{k}}\left|u_{n}(\lambda)-u(\lambda) \| g \exp (-\lambda w)\right| d \lambda \\
& +\int_{r_{k}}^{\infty}\left|u_{n}(\lambda)\left\|g \exp (-\lambda w)\left|d \lambda+\int_{r_{k}}^{\infty}\right| u^{*}(\lambda)\right\| g \exp (-\lambda w)\right| d \lambda \\
& \leqq \frac{\varepsilon_{k}}{\left(r_{k}-\alpha\right) K\left(r_{k}, T\right)}\left(r_{k}-\alpha\right) K\left(r_{k}, T\right)+K_{1} \varepsilon_{k}(t)+\varepsilon_{k}(t)
\end{aligned}
$$

where $K_{1}$ is the factor of the convergence, i.e.

$$
\left|u_{n}(t)\right|<K_{1} \max \{|u(t)| ; 1\}=K_{1}\left|u^{*}(t)\right| .
$$

Thus we obtain

$$
\left|g \mathscr{F}\left(u_{n}-u\right)\right|<\varepsilon_{k}+K^{*} \varepsilon_{k}(t)
$$

for all $t \in[0, T]$ when $n>n_{0}(K)$. This holds for every sequence $\left\{\varepsilon_{k}(t)\right\}_{1}^{\infty}$ so we may assume that $\left|\varepsilon_{k}+K^{*} \varepsilon_{k}(t)\right|<\varepsilon$ for all $t \in[0, T]$. This gives the required result, i.e. $\mathscr{F}$ is $\tau$-continuous.

The proof is now complete.

Part $(b)$ of Theorem 1.7 shows that a large class of endomorphisms can be obtained by integral representation on $H$, but we may have to sacrifice the integral representation on the whole of $M$.

Logarithms. In this part we deal with a certain class of logarithms for which the integral

$$
\int_{a}^{\infty} \exp (-\lambda w) d \lambda
$$

exists for all $\alpha>-\infty$.

L. Máté (8) investigated the so-called bounded logarithms and he gave a necessary and sufficient condition for a logarithm to be bounded. Here we introduce a more restricted class and prove an existence theorem related to the integrals (1.8.1).

Definition 1.5. For every $f, g \in \mathscr{C}$ and for a positive number $\alpha$ we write $\|f\| \leqq \alpha\|g\|$ if $\sup _{t<T}|f(t)| \leqq \sup _{t<T} \alpha|g(t)|$ for every $T>0$. A logarithm is said to be a bounded logarithm if there exists a continuous function $f$ such that, for every $\lambda>0, f . \exp (-\lambda w)$ is a continuous function and

$$
\|f \cdot \exp (-\lambda w)\| \leqq\|f\| .
$$

We say that a logarithm is a bounded logarithm of tauberian type if

$$
\|f \exp (-\lambda w)\| \leqq\|f\| \lambda^{-1-\varepsilon},
$$

$\varepsilon>0$, whenever $\lambda$ is large enough. 
Theorem 1.8. Let $w$ be a bounded logarithm of tauberian type. Then

$$
\mathscr{F}(u)=\int_{0}^{\infty} u(\lambda) \exp (-\lambda w) d \lambda
$$

defines an isomorphism of $\mathrm{H}_{+}$into $M . \dagger$ This isomorphism is $\tau$-continuous and can be uniquely extended to $M$. Moreover there exists no continuous endomorphism $\mathscr{F}_{1}$ of $M$ which is $[H]$-strongly continuous on $H$, different from $\mathscr{F}$ on $H$ and for which $w=\mathscr{F}_{1}(s)$.

Proof. If the integral representation (1.8.2) holds then $\mathscr{F}$ can be uniquely extended from $H_{+}$to $H$ and if $\mathscr{F}$ is $\tau$-continuous then the extension is also $\tau$-continuous. Thus by Theorem $1.6 \mathscr{F}$ can be uniquely extended to a $\tau$ continuous endomorphism of $M$. But $\mathscr{F}_{1}$ is a continuous endomorphism satisfying the condition of Theorem 1.8. Thus by Theorem 1.7, $\mathscr{F}$ has the integral representation (1.7.3) or equivalently (1.8.2).

Now by Theorem 1.7 it is enough to show for $\tau$-convergence that the integral (1.7.1) or (1.8.1) exists for all $\alpha \geqq 0$. Since $w$ is a bounded logarithm of tauberian type,

$$
\begin{array}{r}
\left\|\int_{0}^{\infty} f \exp (-\lambda w) d \lambda\right\| \leqq \int_{0}^{\Lambda_{0}}\|f \exp (-\lambda w)\| d \lambda+\int_{\Lambda_{0}}^{\infty}\|f \exp (-\lambda w)\| d \lambda \\
\leqq\|f\| \int_{0}^{\Lambda_{0}} d \lambda+\|f\| \int_{\Lambda_{0}}^{\infty} \lambda^{-1-\varepsilon} d \lambda<+\infty
\end{array}
$$

This means the integral (1.8.1) is absolutely convergent. The proof is now complete.

Remark 1.9. Let us suppose that $w=1 / k$, where $k$ is a locally integrable function; we can compute the value of the integral (1.8.1). Indeed, by the continuity of the convolution

$$
(w+\mu) \int_{\alpha}^{\beta} e^{-\mu \lambda} f \exp (-\lambda w) d \lambda=\int_{\alpha}^{\beta}(w+\mu) e^{-\mu \lambda} f \cdot \exp (-\lambda w) d \lambda
$$

where $f$ is a continuous function as in Definition 1.5. The right-hand side of this equality can be written in the form $-\int_{\alpha}^{\beta} \frac{d}{d \lambda}\left[f \exp (-\lambda w) e^{-\mu \lambda}\right] d \lambda$. Therefore, because

$$
\left\|\lim _{\beta \rightarrow \infty} e^{-\beta \mu} f \exp (-w \beta)\right\| \leqq\|f\| \lim _{\beta \rightarrow \infty} e^{-\beta \mu}=0
$$

it follows that

$$
\begin{gathered}
\int_{0}^{\infty} e^{-\mu \lambda} f \cdot \exp (-\lambda w) d \lambda=f /(w+\mu) . \\
\dagger H_{+}=\{u: u \in H \text { and spt } u \in[0, \infty)\} \text { where spt } u=\overline{\{x: u(x) \neq 0\}} .
\end{gathered}
$$


Since $w=1 / k$, we obtain

$$
\lim _{\mu \rightarrow 0} \frac{f}{w+\mu}=\lim _{\mu \rightarrow 0} f k\left(1+\sum_{n=1}^{\infty} \mu^{n} k^{n}\right)=f k=f / w .
$$

It is worth noting that if $w=1 / k$, where $k$ is a locally integrable function, the $\mathscr{F}$ map of $s^{-1}=l=\{1\}$ (the operator of integration) is also a locally integrable function.

Now let us consider the value of $\mathscr{F}\left(\left\{\mathscr{H}_{k}(t)\right\}\right)$. By the previous result for $\mathscr{F}(\{1\})=\mathscr{F}(l)$ it is very easy to obtain the value of the integral

$$
\int_{\alpha}^{\infty} \exp (-\lambda w) d \lambda=\mathscr{F}\left(\left\{\mathscr{H}_{\alpha}(t)\right\}\right)
$$

Indeed, $\int_{\alpha}^{\infty}=\int_{\alpha}^{0}+\int_{0}^{\infty}$ and $\exp (-\lambda w)$ is continuous, that is, $\int_{x}^{0}$ exists (see Gesztelyi,(6)); thus

$$
\int_{\alpha}^{\infty} \exp (-\lambda w) d \lambda=\frac{1}{w} \exp (-\alpha w)
$$

Remark 1.10. The exponential function $\exp (-\lambda \sqrt{s})$ is very important for the heat conduction equation. There exists an endomorphism of $M$ for which $\sqrt{s}=\mathscr{F}(s)$. That $\mathscr{F}$ is a $\tau$-continuous endomorphism follows from Theorems 1.7 and 1.8 and from the inequality

Since

$$
0 \leqq e^{-\lambda \sqrt{s}} \leqq 3 \sqrt{\frac{6}{\pi e^{3}}} \frac{1}{\lambda^{2} s} \quad(\lambda>0) .
$$

$$
\left|\int_{0}^{\infty} e^{-\lambda \sqrt{s}} d \lambda\right|=\int_{0}^{\infty} e^{-\lambda \sqrt{s}} d \lambda \leqq \int_{0}^{1} e^{-\lambda \sqrt{s}} d \lambda+1 \int_{1}^{\infty} 3 \sqrt{\frac{6}{\pi e^{3}}} \frac{1}{\lambda^{2}} d \lambda<+\infty
$$

because $e^{-\lambda \sqrt{s}_{s}}$ is a continuous operator function and so $\int_{0}^{1} e^{-\lambda \sqrt{s}_{s}^{-}} d \lambda$ is an operator (see (6)).

It is worth noting how the endomorphism $\mathscr{F}$ acts. Let $x=a / b$ be an arbitrary element of $M ; a, b \in \mathscr{C}$. Let us choose sequences $\left\{p_{n}\right\},\left\{q_{n}\right\}$ in $H$ such that $p_{n} \stackrel{\mathfrak{t}}{\rightarrow} a, q_{n} \stackrel{\mathfrak{x}}{\rightarrow} b$ and let us use their integral formulas which exist by Theorem 1.2. Then

$$
\begin{aligned}
\mathscr{F}\left(q_{n}\right)=\mathscr{F}\left(q_{n}(s)\right)=\mathscr{F}\left(\int_{0}^{\infty} q(\lambda) \exp (-\lambda s) d \lambda\right) \\
=\int_{0}^{\infty} q_{n}(\lambda) \exp (-\lambda \mathscr{F}(s)) d \lambda=\int_{0}^{\infty} q_{n}(\lambda) \exp (-\lambda \sqrt{s}) d \lambda=q_{n}(\sqrt{s}) .
\end{aligned}
$$

Since $\mathscr{F}$ is $\tau$-continuous $q_{n}(\sqrt{s}) \stackrel{M}{\rightarrow} b(\sqrt{s})=$ def $\mathscr{F}(b)$ and

$$
p_{n}(\sqrt{s}) \stackrel{M}{\rightarrow} a(\sqrt{s})={ }_{\mathrm{def}} \mathscr{F}(a) .
$$


But

and then

$$
\mathscr{F}\left(p_{n} / q_{n}\right) \stackrel{M}{\rightarrow} \mathscr{F}(a / b)=\mathscr{F}(x)
$$

$$
\frac{\mathscr{F}\left(p_{n}\right)}{\mathscr{F}\left(q_{n}\right)}=\frac{p_{n}(\sqrt{s})}{q_{n}(\sqrt{s})} \stackrel{M}{\rightarrow} \mathscr{F}\left(\frac{a}{b}\right)=\mathscr{F}(x)=x(\sqrt{s}) .
$$

The above reasoning holds for every $\tau$-continuous endomorphism with a modification $w=\mathscr{F}(s)$ instead of $\sqrt{s}$. (More generally it holds for pointwise continuous endomorphisms which have integral representations on $H$.)

Now we shall consider another class of logarithms which is closer to the classical Efros transformation. (See Bleyer (1), Efros (4) and Ditkin \& Prudnikov, (3)). $\dagger$

Definition 1.6.1. A logarithm $w$ is called Laplace transformable in the sense of Ditkin if there are functions $f$ and $g$ in $\mathscr{L}$ (the set of Laplace transformable functions for which the Laplace integral converges absolutely in some half-plane $\operatorname{Re} p>\sigma_{f}$ ) such that $w=f / g$. In this case the Laplace transform of $w$ is defined by

$$
\mathscr{L}_{D}(w)=\frac{\mathscr{L}(f)}{\mathscr{L}(g)}
$$

for every $\operatorname{Re} p \geqq \max \left(\sigma_{f}, \sigma_{g}\right)=\sigma_{w^{*}} \quad$ (See (3).)

(Not every logarithm is Laplace transformable, e.g. $w=\left\{e^{t^{k}}\right\}$ for $k=2,3, \ldots$ is a bounded logarithm but not Laplace transformable, see Stopp (11).)

E. Gesztelyi discussed Laplace transformable logarithms and he gave a more general definition of them.

Definition 1.6.2. A logarithm $w$ is Laplace transformable according to Gesztelyi if the limit

$$
M^{\mathrm{II}}-\lim _{n \rightarrow \infty} U_{n} T^{-p_{W}}=\mathscr{L}_{G}(w)=w^{*}(p)
$$

exists for some complex number $p$.

Here we refer to Gesztelyi (7). The definitions of $U_{n}, T^{\alpha}$ are as follows: $U_{n}(f)=\{n f(n t)\}, T^{\alpha}(f)=\left\{e^{\alpha t} f(t)\right\}$ if $f \in \mathscr{C}$ and

if $x=a / b \in M$ where $a, b \in \mathscr{C}$.

$$
U_{n}(x)=U_{n}\left(\frac{a}{b}\right)=\frac{U_{n}(a)}{U_{n}(b)}, \quad T^{\alpha}(x)=T^{\alpha}\left(\frac{a}{b}\right)=\frac{T^{\alpha}(a)}{T^{\alpha}(b)}
$$

$\dagger$ A linear function transformation $E$ defined for Laplace transformable functions (here we mean the Laplace integral of a function is absolutely convergent) is called an Efrostransformation if it is of the form

where

$$
E f(t)=\int_{0}^{\infty} k(x, t) f(x) d x
$$

$$
\int_{0}^{\infty} k(x, t) \exp (-p t) d t=\bar{a}(p) e^{-\dot{x}(p)}
$$

if $\operatorname{Re} p>\sigma_{c}$ and $\operatorname{Re} g(p)>\sigma_{c}$ and we suppose that the last integral absolutely converges for all $x \in[0, \infty)$. Writing the kernel function of $(E)$ in operator terms

$$
\{k(x, t)\}=\hat{a}(s) \exp (-x \bar{q}(s)) \text {. }
$$




$$
M^{\mathrm{II}}-\lim _{n \rightarrow \infty} x_{n}=x \text { if } x_{n}=a_{n} / b_{n}, x^{\prime}=a / b \text { and } a_{n} \rightarrow a, b_{n} \rightarrow b
$$

almost uniformly in $[0, \infty)$.

It is obvious that $M$-convergence implies $M^{\mathrm{II}}$-convergence.

E. Gesztelyi proved that if $\mathscr{L}_{D}(w)$ exists in a certain domain then $\mathscr{L}_{G}(w)$ also exists there and $\mathscr{L}_{D}(w)=\mathscr{L}_{G}(w)$.

Now we prove a lemma related to an exponential function generated by a Laplace transformable logarithm.

Lemma 1.11. If $\mathscr{I}(\lambda)=\exp (-\lambda w)$ and $w$ is a Laplace transformable logarithm in the sense of Ditkin then $\mathscr{I}(\lambda)$ is a Laplace transformable operator function in the sense of Gesztelyi and

$$
\mathscr{L}_{G}(\mathscr{A}(\lambda))=\exp \left(-\lambda w^{*}(p)\right)
$$

for every $\operatorname{Re} p>\sigma_{w}$ where $\mathscr{L}_{D}(w)=\mathscr{L}_{G}(w)=w^{*}(p)$ for all $\operatorname{Re} p>\sigma_{\omega}$.

Proof. Indeed, using Gesztelyi's method, there is only one thing to prove, namely that

$$
U_{n} T^{-p}(\exp (-\lambda w))=\exp \left(-\lambda U_{n} T^{-p} w\right)
$$

for every $\operatorname{Re} p>\sigma_{w}$. Since the exponential function is continuous and the operator transformations $U_{n}$ and $T^{-p}$ are continuous endomorphisms of $M$ (see Gesztelyi (7)) the lemma obviously follows from (1.11.1).

To prove (1.11.1), note that if $f(\lambda)$ is an arbitrary differentiable operator function then

and

$$
\frac{\partial}{\partial \lambda} U_{n}(f(\lambda))=U_{n}\left(\frac{\partial f(\lambda)}{\partial \lambda}\right)
$$

Thus

$$
\frac{\partial}{\partial \lambda} T^{-p}(f(\lambda))=T^{-p}\left(\frac{\partial f(\lambda)}{\partial \lambda}\right)
$$

$$
\frac{\partial}{\partial \lambda} U_{n}(\exp (-\lambda w))=U_{n}(-w \exp (-\lambda w))=-U_{n}(w) U_{n}(\exp (-\lambda w)) .
$$

Further, the definition of the exponential function implies that $U_{n}(\exp (-\lambda w))$ is a solution of the operator differential equation

$$
x^{\prime}(\lambda)+U_{n}(w) x(\lambda)=0 .
$$

A similar result can be obtained for $T^{-p}(w)$.

Now (1.11.1) evidently follows from the uniqueness of the solution of (1.11.2).

Let us remark that the fact that $w=f / g$ and $f, g$ are in $\mathscr{L}$ does not imply that $\exp (-\lambda w)=\tau(\lambda) / g_{1}$ where $g_{1} \in \mathscr{L}$ and $\tau(\lambda)=\{\tau(\lambda, t)\}$ is an (absolutely) 
Laplace transformable function in the variable $t$, i.e. $\exp (-\lambda w)$ may not be a Laplace transformable operator function in the sense of Ditkin.

Theorem 1.12. Every (classical) Efros-transformation can be extended to a $\tau$-continuous linear operator transformation of the Mikusinski operator field and this extension is unique.

Proof. Using the properties of classical Efros-transformations we obtain an operator $a$ in $M$ (in fact, it is a Laplace transformable operator in Ditkin's sense) for the Efros transformation $E$ such that $a^{-1} E$ is an isomorphism of $\mathscr{L}$ into $M$. This operator always exists, as can be seen from the connection between the operator and the Laplace transformation forms of the Efros transformation. (See the definition of the Efros transformation, Bleyer (1) and Efros (4).) $\dagger$

Now we prove that $a^{-1} E$, or equivalently $E$, is $\tau$-continuous on $\mathscr{L}(H \subset \mathscr{L}$ is obvious). Indeed, if $u_{n} \stackrel{\dot{x}}{\rightarrow} u$ and $u_{n}, u$ are Laplace transformable functions then there exists a Laplace transformable function $u^{*}$ such that $\left|u_{n}\right|<\left|u^{*}\right|$ and $|u|<\left|u^{*}\right|$. Thus $\mathscr{L}_{D}\left(u_{n}\right) \rightarrow \mathscr{F}_{D}(u)$ uniformly in the half plane $\operatorname{Re} p \geqq \sigma_{0}$ and from this and from the Theorem of Efros we deduce that $\mathscr{L}_{D}\left(E u_{n}\right) \rightarrow \mathscr{L}_{D}(E u)$ uniformly in $\operatorname{Re} p \geqq \sigma_{0}$. Finally this implies the desired convergence $E u_{n} \rightarrow E u$.

Several classical integral transformations can be identified as Efros transformations. Thus they can be extended uniquely to $\tau$-continuous linear operator transformations of the whole operator field. For example, the Hankel transformation belongs to the above class, as do several types of integral transformations based on special functions.

\section{Pointwise continuous endomorphisms and their integral representations}

In this part we shall again deal with pointwise continuous endomorphisms of $M$. We know by Theorem 1.6 that they can be extended uniquely to the whole of $M$; that is, they are completely characterized on $H$. The purpose of this part is to give a generalization of the theorem, due to $E$. Gesztelyi (7), by which every strongly continuous endomorphism $\mathscr{F}$ of $M$ has an integral representation on $\mathscr{C} \mathscr{U}, \mathscr{F}(s)$ is a logarithm and

for every $u \in \mathscr{C} \mathscr{U}$.

$$
\mathscr{F}(u)=\int_{-\infty}^{\infty} u(\lambda) \exp (-\lambda \mathscr{F}(s)) d \lambda
$$
(6).

The integral is understood to be a Stieltjes type integral defined by Gesztelyi

† Theorem of Efros. Let $f(x)$ be an absolutely Laplace transformable function on the half plane $\operatorname{Re} p>0$, then

$$
\mathscr{L}(E f)=\bar{a}(p) f(\bar{q}(p))
$$

for $\operatorname{Re} p>\sigma$, where $f(p)=\mathscr{L}(f)$. (See the definition of the Efros transform in a previous footnote). Here we refer to Efros (4) and Ditkin \& Prudinkov (3). 
Theorem 2.1. If the operator function $f(\lambda)$ is integrable with respect to $\phi(\lambda)$ $(\phi(\lambda)$ is a numerical function) on $[\alpha, \beta]$ then the operator function $\mathscr{F}(f(\lambda))=g(\lambda)$ is also. Moreover

$$
\int_{\alpha}^{\beta} \mathscr{F}(f(\lambda)) d \phi(\lambda)=\mathscr{F}\left(\int_{\alpha}^{\beta} f(\lambda) d \phi(\lambda)\right) .
$$

Proof. (Our proof follows that of the corresponding theorem of Gesztelyi (7).) According to the definition of the integral

$$
\lim a_{n}=\lim _{n \rightarrow \infty} \sum_{k=1}^{n} f\left(\xi_{k}^{(n)}\right)\left[\phi\left(\hat{\lambda}_{k}^{(n)}\right)-\phi\left(\lambda_{k-1}^{(n)}\right)\right]=\int_{\alpha}^{\beta} f(\lambda) d \phi(\lambda)
$$

independent of the choice of numbers

$$
\begin{aligned}
& \alpha_{0}=\lambda_{0}^{(n)}<\lambda_{1}^{(n)}<\ldots<\lambda_{n}^{(n)}=\beta(n=1,2, \ldots), \\
& \lambda_{k-1}^{(n)} \leqq \xi_{k}^{(n)} \leqq \lambda_{k}^{(n)} \quad(k=1,2, \ldots, n)
\end{aligned}
$$

provided that $\lim _{n \rightarrow \infty} \max _{k=1,2, \ldots, n}\left(\lambda_{k}^{(n)}-\lambda_{k-1}^{(n)}\right)=0 . \quad$ By the linearity of $\mathscr{F}$ $b_{n}=\sum_{k=1}^{n} \mathscr{F}\left(f\left(\xi_{k}^{(n)}\right)\right)\left[\phi\left(\lambda_{k}^{(n)}\right)-\phi\left(\lambda_{k-1}^{(n)}\right)\right]$

Now we obtain

$$
=\mathscr{F}\left(\sum_{k=1}^{n} f\left(\xi_{k}^{(n)}\right)\left[\phi\left(\lambda_{k}^{(n)}\right)-\phi\left(\lambda_{k-1}^{(n)}\right)\right]\right)=\mathscr{F}\left(a_{n}\right) .
$$

$$
\mathscr{F}\left(a_{n}\right) \stackrel{M}{\rightarrow} \mathscr{F}\left(\int_{\alpha}^{\beta} f(\lambda) d \phi(\lambda)\right) \text { as } n \rightarrow \infty
$$

by the continuity of $\mathscr{F}$. The proof is complete.

Theorem 2.2. If $\int_{-\infty}^{\infty} f(\lambda) d \phi(\lambda)$ exists then so does $\int_{-\infty}^{\infty} \mathscr{F}(f(\lambda)) d \phi(\lambda)$ and

$$
\int_{-\infty}^{\infty} \mathscr{F}(f(\lambda)) d \phi(\lambda)=\mathscr{F}\left(\int_{-\infty}^{\infty} f(\lambda) d \phi(\lambda)\right) .
$$

Proof. The truth of the statement follows from the continuity of $\mathscr{F}$ and from the relation

$$
\int_{a_{n}}^{b_{n}} f(\lambda) d \phi(\lambda) \stackrel{M}{\rightarrow} \int_{-\infty}^{\infty} f(\lambda) d \phi(\lambda)
$$

as $a_{n} \rightarrow-\infty, b_{n} \rightarrow \infty$ in any manner.

Theorem 2.3. If $\mathscr{F}$ is a pointwise continuous linear transformation then

for all $u \in \mathscr{C} \mathscr{U}$.

$$
\mathscr{F}(u)=\int_{-\infty}^{\infty} u(\lambda) \mathscr{F}(\exp (-\lambda s)) d \lambda
$$


Proof. The theorem obviously follows from Theorems 1.2 and 2.2 and from the relation

$$
\mathscr{F}(u(\lambda) \exp (-\lambda s))=u(\lambda) \mathscr{F}(\exp (-\lambda s))
$$

For the integral representation of pointwise continuous endomorphisms we will need some new definitions.

Definition 2.1. Let $f(\lambda)$ be an operator-valued function of a real variable $\lambda$. We call $f(\lambda)$ (sequentially) continuous if $\lim f\left(\lambda_{n}\right)=f\left(\lambda_{0}\right)$ whenever $\lambda_{n} \rightarrow \lambda_{0}$.

Definition 2.2. $f(\lambda)$ is called (sequentially) differentiable at a point $\lambda_{0}$ with derivative $f^{\prime}\left(\lambda_{0}\right)$ if

whenever $\lambda_{n} \rightarrow \lambda_{0}$.

$$
\lim \frac{f\left(\lambda_{n}\right)-f\left(\lambda_{0}\right)}{\lambda_{n}-\lambda_{0}}=f^{\prime}\left(\lambda_{0}\right)
$$

It is easy to see that every operator function which is continuous or differentiable in Mikusinśki's sense is continuous or differentiable in the sense of Definitions 2.1 and 2.2. The converse of this is probably not true.

Lemma 2.4. If $f(\lambda)$ is differentiable in the sense of Definition 2.2 then it is continuous in the sense of Definition 2.1.

Proof. This follows from

because

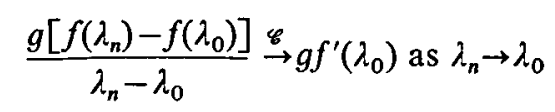

$$
g\left[f\left(\lambda_{n}\right)-f\left(\lambda_{0}\right)\right]=\left(\lambda_{n}-\lambda_{0}\right)\left[g f^{\prime}\left(\lambda_{0}\right)+\{\varepsilon(t)\}\right] \stackrel{\mathscr{\varphi}}{\rightarrow} 0
$$

where $\varepsilon(t)$ is a continuous function. Here $g$ and $\varepsilon(t)$ may depend on the sequence $\left\{\lambda_{n}\right\}$.

The previous remarks and Lemma 2.4 show that the notions of sequential continuity and differentiation are weaker than Mikusinśki's notions but they are consistent with Mikusinski's definitions.

Definition 2.3. Let us consider the functional equation

$$
E(x+y) E(0)=E(x) E(y)
$$

for the operator function $E(x)$ in some real interval containing 0 . Let $E(x)$ be a sequentially differentiable solution of $(D .2 .3 .1)$ for which $E^{\prime}(0)=-w E(0)$. This solution is said to be a weakly exponential function and the operator $w$ is called a weak logarithm.

Lemma 2.5. Every exponential function is a weakly exponential function and every logarithm is a weak logarithm.

Proof. It is well-known that every exponential function satisfies the equation (D.2.3.1) and $[\exp (-\lambda w)]^{\prime}=-w \exp (-\lambda w)$ also holds. Thus, by Lemma 2.4 , Lemma 2.5 follows immediately. 
Theorem 2.6. Every weakly exponential function $E(x)$ has the following properties:

(a) it can be defined for every real $x$;

(b) $E\left(x_{0}\right)=0$, for some $x_{0}$, implies $E(x)=0$ for all real $x$;

(c) $\frac{d E(x)}{d x}+w E(x)=0$

where differentiation should be taken in the sense of Definition 2.2;

(d) $E(x)$ is infinitely differentiable;

(e) the equation (D.2.3.1) has only one solution for which $E(0)=1$, $E^{\prime}(0)=-w$ and $E(x)=\mathscr{F}(\exp (-x s))$ where $\mathscr{F}$ is a pointwise continuous endomorphism of the operator field $M$.

Proof. (a) $\dagger$ Let $E(x)$ be a non-trivial solution of $(D .2 .3 .1)$ in the interval $x_{1} \leqq x \leqq x_{2}$. Then there is $x_{0} \in\left(x_{1}, x_{2}\right)$ such that $E\left(x_{0}\right) \neq 0$. For, if $E(x)=0$ for every $x_{1}<x<x_{2}$ then by continuity, $E\left(x_{1}\right)=E\left(x_{2}\right)=0$ would follow. Let $x_{0} \in\left(x_{1}, x_{2}\right)$ be such that $E\left(x_{0}\right)=a_{0} \neq 0$. Then $E(x)$ can be extended beyond the interval $\left[x_{1}, x_{2}\right]$ using (D.2.3.1). Set $x_{3}=2 x_{2}-x_{0}>x_{2}$ and

$$
E_{1}(x)=a_{0}^{-1} E\left(x_{2}\right) E\left(x-x_{2}+x_{0}\right)
$$

for $x_{2}<x<x_{3}$. Since $x-x_{2}+x_{0} \in\left[x_{1}, x_{2}\right]$ we obtain that $E_{1}(x)$ is continuous in $\left[x_{2}, x_{3}\right]$ and $E_{1}\left(x_{2}\right)=E\left(x_{2}\right)$. This means that $E_{1}(x)$ is a continuous extension of $E(x)$. This method can be used in both directions and the interval $\left[x_{1}, x_{2}\right]$ is extended by $\left|x_{2}-x_{0}\right|$ to the right, by $\left|x_{0}-x_{1}\right|$ to the left. This means that every real $x$ can be reached by a finite number of such extensions.

(b) If $E\left(x_{0}\right)=0$ then $E(x) E\left(x-x_{0}\right)=E(0) E(x)=0$. Thus either $E(x)=0$ or $E(0)=0$. Put $y=x$ in (D.2.3.1). Then $E(x) E(x)=E(0) E(2 x)=0$ implying $E(x)=0$.

(c) $E(x)$ is a sequentially differentiable solution of (D.2.3.1) by Definition 2.3. Now

$$
\begin{aligned}
\frac{d E(x)}{d x}=\lim _{x_{n} \rightarrow x} \frac{E\left(x_{n}\right)-E(x)}{x_{n}-x}=\lim _{x_{n} \rightarrow x} \frac{E(x)}{E(0)} & \frac{E\left(x_{n}-x\right)-E(0)}{x_{n}-x} \\
& =\frac{E(x)}{E(0)} \lim _{x_{n} \rightarrow x} \frac{E\left(x_{n}-x\right)-E(0)}{x_{n}-x}=\frac{E(x)}{E(0)} E^{\prime}(0)
\end{aligned}
$$

and if we set $-E(0) w=E^{\prime}(0)$ we obtain an equation of type (2.6.1) for the weakly exponential function $E(x)$.

(d) This is obvious since $E(x)$ satisfies the equation (2.6.1).

(e) If $E(x)=\mathscr{F}(\exp (-x s))$ then $E(x)$ is a solution (D.2.3.1) whenever $\mathscr{F}$ is a pointwise continuous endomorphism. Since in that case differentiation

† See A. Erdélyi (5), J. Mikusinśki (9). 
with respect to the parameter $x$ and application of $\mathscr{F}$ commute, we have $E^{\prime}(0)=-\mathscr{F}(s)$ and $E(0)=\mathscr{F}(1)=1$. Moreover, by the continuity of $\mathscr{F}$ and by the well-known limit

we obtain

$$
(1+x s / n)^{-n} \stackrel{M}{\rightarrow} \exp (-x s) \text { as } n \rightarrow \infty
$$

$$
\mathscr{F}\left[(1+x s / n)^{-n}\right] \stackrel{M}{\rightarrow} \mathscr{F}(\exp (-x s)) \text { as } n \rightarrow \infty \text {. }
$$

Now we prove that $E(x)$ is the unique solution of (D.2.3.1) under the conditions $E(0)=1, E^{\prime}(0)=-\mathscr{F}(s)=-w$ and $E(x)=\mathscr{F}(\exp (-x s))$ where $\mathscr{F}$ is a pointwise continuous endomorphism. Assuming that there are two different solutions $E_{1}, E_{2}$ satisfying the above conditions we have by (2.6.2)

$$
\begin{aligned}
E_{1}(x) & =\lim _{n \rightarrow \infty} \mathscr{F}_{1}\left[(1+x s / n)^{-n}\right]=\lim _{n \rightarrow \infty}\left[\mathscr{F}_{1}\left(P_{n}(x s)\right)\right]^{-1}=\lim _{n \rightarrow \infty}\left[P_{n}\left(x \mathscr{F}_{1}(s)\right)\right]^{-1} \\
& =\lim _{n \rightarrow \infty}\left[P_{n}(x w)\right]^{-1}=\lim _{n \rightarrow \infty}\left[P_{n}\left(x \mathscr{F}_{2}(s)\right)\right]^{-1}=\lim _{n \rightarrow \infty}\left[\mathscr{F}_{2}\left(P_{n}(x s)\right)\right]^{-1} \\
& =\lim _{n \rightarrow \infty} \mathscr{F}_{2}\left[(1+x s / n)^{-n}\right]=E_{2}(x)
\end{aligned}
$$

where $P_{n}(\cdot)$ is a polynomial.

The proof of the theorem is now complete.

Now we give a list of some further properties of the weakly exponential function which can be obtained by simple computations.

$$
\text { If } \frac{E_{1}^{\prime}(0)}{E_{1}(0)}=-w \text { and } \frac{E_{2}^{\prime}(0)}{E_{2}(0)}=-w \text { then }\left(\frac{E_{1}(x)}{E_{2}(x)}\right)^{\prime}=0 \text {. }
$$

Indeed, it follows from (2.6.1) that

for all $x$.

$$
E_{1}^{\prime}(x) E_{2}(x)-E_{2}^{\prime}(x) E_{1}(x) \equiv 0
$$

(2.6.4) If $E(x)$ is a weakly exponential function such that $E(0)=c \neq 0$ and $E^{\prime}(0)=-w c \neq 0$ then there is a weakly exponential function $E_{1}(x)$ for which $E_{1}^{\prime}(x)=E(x)$.

Indeed, $E_{1}(x)=\frac{c}{E^{\prime}(0)} E(x)$ satisfies the conditions and $E_{1}(x)$ is a sequentially differentiable solution of (D.2.3.1).

(2.6.5) A non-constant weakly exponential function cannot be written in the form $E_{1}(x)+c$ where $E_{1}(x)$ is a weakly exponential function and $c$ is a non-zero constant.

Let us assume that $E(x)=E_{1}(x)+c$ then

$$
\left(E_{1}(x+y)+c\right)\left(E_{1}(0)+c\right)=\left(E_{1}(x)+c\right)\left(E_{1}(y)+c\right)
$$

and since $E_{1}(x)$ is a solution of (D.2.3.1) we obtain

$$
c\left(E_{1}(0)+E_{1}(x+y)\right)=c\left(E_{1}(x)+E_{1}(y)\right) .
$$


Taking $x=y$ and multiplying by $E_{1}(0) \neq 0$ (by virtue of Theorem 2.6b) we have

$$
c\left(E_{1}^{2}(0)-2 E_{1}(x) E_{1}(0)+E_{1}(2 x) E_{1}(0)\right)=0
$$

and as $E_{1}(2 x) E_{1}(0)=E_{1}(x) E_{1}(x)$ by (D.2.3.1), $c\left(E_{1}(0)-E_{1}(x)\right)^{2}=0$ follows. Thus either $c=0$ or $E_{1}(0)=E_{1}(x)$ for every real $x$.

(2.6.6) If $E(x) \neq 0$ is a weakly exponential function and if there exists a weakly exponential function $E_{1}(x)$ for which $E_{1}^{\prime}(x)=E(x)$ then $E^{\prime}(0) \neq 0$.

If $E_{1}^{\prime}(x)=E(x)$ then $E_{1}^{\prime}(0)=E(0) \neq 0$. Now

Hence obviously

$$
E^{\prime}(x)=E_{1}^{\prime \prime}(x)=\left(E_{1}(x) \frac{E_{1}^{\prime}(0)}{E_{1}(0)}\right)^{\prime}=\frac{E_{1}^{\prime}(x)}{E_{1}(0)} E_{1}^{\prime}(0) \text {. }
$$

$$
E^{\prime}(0)=\frac{\left(E_{1}^{\prime}(0)\right)^{2}}{E_{1}(0)}=\frac{E^{2}(0)}{E_{1}(0)} \neq 0
$$

follows, since $E_{1}(0) \neq 0$ by Theorem $2.6(b)$. Thus $E^{\prime}(x)=E(x) E^{\prime}(0) \neq 0$ for all $x$.

If we could prove that $f^{\prime}(x)=0$ (in the sense of Definition 2.2) implies $f(x)=c$ or the weaker statement that $f^{\prime}(x)=0$ and $f(x)$ is a weakly exponential function implies $f(x)=c$ then the uniqueness of the common solution of (2.6.1) and (D.2.3.1) would follow from (2.6.3) or (2.6.6). The first of these does not seem to be true and if the second statement is valid it is probably very hard to prove.

Notation. As we know, from Theorem 2.6(e), that there is a unique weakly exponential function which is a solution of (D.2.3.1) under the conditions of Theorem 2.6(e) we shall write $\operatorname{Exp}(-x \omega)$ instead of $E(x)$.

Theorem 2.7. Let $\mathscr{F}$ be a pointwise continuous endomorphism of $M$. Then $\mathscr{F}(s)$ is a weak logarithm and

for every $u \in \mathscr{C} \mathscr{U}$.

$$
\mathscr{F}(u)=\int_{-\infty}^{\infty} u(\lambda) \operatorname{Exp}(-\lambda \mathscr{F}(s)) d \lambda
$$

Proof. $E(\lambda)=\mathscr{F}(\exp (-\lambda s))$ is a common solution of (D.2.3.1) and (2.6.1) by the pointwise continuity of $\mathscr{F}$. By the simple computation

$$
\mathscr{F}(s)=-\frac{E^{\prime}(0)}{E(0)}=\left.(\mathscr{F}(\exp (-\lambda s)))^{\prime}\right|_{\lambda=0}
$$

we obtain that $\mathscr{F}(s)$ is a weak logarithm. By Theorems 2.3 and 2.6(e) the statement of Theorem 2.7 follows from

$$
\mathscr{P}(\exp (-\lambda s))=E(x)=\operatorname{Exp}(-\lambda \mathscr{F}(s)) \text {. }
$$

(See the above note on Notation.) 
Theorem 2.8. If $\mathscr{F}$ is a pointwise continuous endomorphism of $M$ and $\mathscr{F}(s)$ is a Mikusinśki logarithm then

for all $u \in \mathscr{C} \mathscr{U}$.

$$
\int_{-\infty}^{\infty} u(\lambda) \exp (-\lambda \mathscr{F}(s)) d \lambda=\mathscr{F}(u)
$$

Proof. By virtue of Theorem 2.6(e), $\mathscr{F}(\exp (-\lambda s))$ is the unique solution of (D.2.3.1) with $\mathscr{F}(s)=-w$. So by Theorem 2.4 the existence of the integral representation (2.8.1) follows from

$$
\mathscr{F}(\exp (-\lambda s))=\operatorname{Exp}(-\lambda \mathscr{F}(s))=\exp (-\lambda \mathscr{F}(s))
$$

Let us remark that Gesztelyi's representation theorem of strongly continuous endomorphisms now immediately follows from Theorems 1.1, 1.5 and 2.7. Theorem 1.1 proves the pointwise continuity. Theorem 1.5 proves that the map of $s$ is a logarithm, and finally from Theorem 2.7 we have the required integral representation.

\section{REFERENCES}

(1) A. BLEYer, On continuous endomorphisms of Mikusinśki's operator field, Acta Math. Acad. Sci. Hungar. 21 (3-4) (1970), 393-402.

(2) T. K. BоEнme, On sequences of continuous functions and convolution, Studia Math. 25 (1965), 333-335.

(3) V. A. Ditkin and A. P. PRUdinkov, Integral transforms and operational calculus (Pergamon Press, 1965).

(4) A. M. Efros, On some applications of operational calculus to analysis (in Russian), Mat. Sb. 42 (1935).

(5) A. ERDÉly, Operational calculus and generalized functions (New York, 1963).

(6) E. GeszTelyI, Über das Stieltjes-Integral von Operatorfunktionen II, Publ. Math. Debrecen 13 (1966), 313-324.

(7) E. GeszTely1, Über lineare Operatortransformation, Publ. Math. Debrecen 14 (1967), 169-206.

(8) L. MátÉ Note on Mikusinśki's logarithm, Bull. Acad. Polon. Sci. Ser. Sci. Math. 13 (1965), 641-644.

(9) J. MikusinśKI, Operational calculus (New York, 1959).

(10) J. Mrkusinśkı, An approximation theorem and its application, Studia Math. 27 (1966), 141-145.

(11) F. STOPP, Zur funktiontheoretischen Auffassung der Mikusinśkischen Operatorenrechnung, Math. Nachr. 32 (1966), 187-205.

BUDAPEST

HUNGARY 